\title{
NEW CAPTURE GAMMA-RAY LIBRARY AND ATLAS OF SPECTRA FOR ALL ELEMENTS
}

\author{
R. B. FIRESTONE \\ Lawrence Berkeley National Laboratory, \\ Berkeley, CA 94720, USA \\ E-mail: rbf@lbl.gov \\ ZS. REVAY AND G. L. MOLNAR \\ Institute of Isotope and Surface Chemistry, \\ Chemical Research Center, Budapest, H-1525, Hungary \\ E-mail: molnar@alpha0.iki.kfki.hu
}

\begin{abstract}
A new library comprising 30 thousand neutron capture gamma rays has been created by combining new measurements on natural elements from Budapest and literature data for all stable isotope targets. All energies and intensities are consistent in that they are based on the chlorine and nitrogen standards, respectively. Accurate neutron binding energies and thermal capture cross-sections could also be inferred for all cases where the level scheme is sufficiently complete. The new data can be used for nuclear structure investigations, reaction model calculations, and a number of applications, such as Prompt Gamma-ray Activation Analysis (PGAA).
\end{abstract}

\section{Introduction}

The development of guided neutron beams has lead to renewed interest in capture gamma-ray spectroscopy for nuclear structure investigations and applications including Prompt Gamma-ray Activation Analysis (PGAA). Until now, the most comprehensive compilation of capture gamma-ray data was published by Lone et al [1] based on $\mathrm{Ge}(\mathrm{Li})$ measurements of elemental spectra by Rasmussen [2] in the 1960's. These data give gamma-ray yields per 100 neutron captures, but lack isotopic assignments and statistical uncertainties. The gamma rays were not compared with nuclear structure information resulting in many incorrect assignments.

Recently, new capture gamma-ray measurements for all elements from Hydrogen to Uranium have been performed with the guided neutron beam at the Budapest Reactor [3,4]. Stoichiometric relationships in various compounds were used to determine all gamma-ray cross sections relative to 
the chlorine and nitrogen standards. In 1998 an International Atomic Energy Agency Coordinated Research Project (IAEA CRP) [5] was formed to evaluate the Budapest data and other information from the literature and create a comprehensive database for PGAA. This database of $\approx 30,000$ gamma-rays from prompt neutron capture has now been completed.

\section{Nuclear Data Sources}

Data from the compilation of Lone et al [1] were not used in this evaluation except for comparison with the final results. A principal source of evaluated capture gamma-ray data is the Evaluated Nuclear Structure Data File (ENSDF) [6]. ENSDF is compiled by an international collaboration of evaluators under the auspices of the IAEA Nuclear Structure and Decay Data (NSDD) Working group. The data are organized by isotope and the gamma-ray intensities are normalized per 100 neutron captures. However, the primary emphasis of ENSDF has been to derive nuclear structure information and the gamma-ray intensity normalizations are sometimes incomplete or missing. In this work the ENSDF normalizations were checked, modified, and additional data incorporated from the ENSDF Adopted datasets when applicable. Cut-off dates for the ENSDF evaluations vary, so we have updated the data as necessary from more recent references found in the Nuclear Science Reference file (NSR) [7].

Reedy et al [8] have evaluated capture gamma-ray data for the isotopes of elements with $Z=1-30$. Gamma-ray intensities were also normalized per 100 neutron captures. The literature covered by this compilation is generally the same as ENSDF, but the data have been evaluated for applied use. We have substituted the Reedy et al datasets for ENSDF whenever they were deemed superior.

The Budapest Reactor gamma-ray data were measured for each element, and energies and elemental cross sections were provided. Isotopic assignments were derived by comparison of the energies and relative intensities to those in ENSDF. These data were then compiled into ENSDF format for comparison with the literature data.

Other data sources used in this evaluation include isotopic abundances from Rosman and Taylor [9], total thermal neutron cross sections from Mughabghab et al [10], Westcott g-factors from Holden [11], IUPAC atomic weights [12], and neutron separation energies from Audi et al [13]. 


\section{Evaluation Methodology}

Two source datasets were constructed using ENSDF formats. A literature source was constructed from ENSDF, Reedy et al, and/or original papers. This dataset was edited for consistency and completeness, and the gammaray intensity normalizations were checked by comparison with the intensity balance through the level scheme. Additional data, including conversion coefficients and weak gamma-ray branches were added from the ENSDF Adopted Levels, Gammas dataset. The second dataset was constructed from the Budapest Reactor data as described above. In some cases no adequate literature data had been published and only the Budapest Reactor data were used.

\subsection{Adopted Gamma-ray Energies}

Adopted gamma-ray energies were averaged by a weighted least-squares fit of the measured gamma-ray energies to the level scheme. Data from both sets were analyzed simultaneously and, as the gamma energies represent the differences in the well determined level energies, even weak transitions could typically be determined to better precision. A chi-squared analysis of the fit was performed, and the uncertainties of individual outliers with $\chi^{2} / f>4$, and all data for sets with $\chi^{2} / f>1$ were increased and the fit repeated until $\chi^{2} / f=1$.

\subsection{Adopted Gamma-ray Production Cross Sections}

The Budapest Reactor gamma-ray intensity data were reported as elemental cross sections, and the literature intensities were generally compiled per 100 neutron captures of the isotope. These data were averaged by one of two methods. If a well determined gamma-ray cross section existed in the literature, the gamma-ray intensities in the literature dataset were renormalized with that value and directly weighted averaged with the Budapest values. For most cases no accurate normalization existed, and the literature values were renormalized by a factor chosen to minimize the weighted average difference between the literature and Budapest intensity data. The renormalized intensity data were then weighted averaged with the Budapest data to obtain the adopted cross sections. A chi-squared analysis was performed, as described for the energies, to handle individual outliers and discrepant datasets. In addition, the skew in the chi-squared distribution, as a function of energy, was used to determine systematic differences in the underlying efficiency curves, and discrepant data were removed from the analysis. 
The gamma-ray intensity balance through the level scheme was used to determine the quality and completeness of the evaluated data. The sum of gamma-ray cross sections feeding the ground state could be compared with the values from Mughabghab et al [10], and the ratio of the sum of primary gamma-ray cross sections to the ground state feedings give an indication of the completeness of the data. Intensity balances in or out of intermediary levels also indicate missing or anomalous intensity. An example of the intensity balance for the adopted data for ${ }^{24} \mathrm{Mg}(n, \gamma)$ is shown in Table 1. No important discrepancies were noted in this case.

Table 1. Gamma-ray cross section balance for ${ }^{24} \mathrm{Mg}(n, \gamma)$.

\begin{tabular}{|c|c|c|c|}
\hline Level Energy & $\sigma($ In $)$ & $\sigma($ Out $)$ & $\Delta \sigma$ \\
\hline 0.0 & $0.0536(14)$ & 0.0 & $0.0536(14)$ \\
585.01 & $0.0406(11)$ & $0.0398(14)$ & $-0.0008(18)$ \\
974.68 & $0.0157(4)$ & $0.0158(4)$ & 0.0 \\
1964.69 & $0.00022(2)$ & $0.00026(3)$ & $0.00004(4)$ \\
2563.35 & $0.00202(10)$ & $0.00179(7)$ & $0.00023(12)$ \\
2801.54 & $0.00047(4)$ & $0.00061(5)$ & $0.00013(6)$ \\
3413.35 & $0.0411(14)$ & $0.0416(11)$ & 0.0 \\
4276.33 & $0.0105(4)$ & $0.0107(3)$ & $0.00002(5)$ \\
4358.2 & $0.000009(23)$ & 0.0 & $-0.00009(23)$ \\
5116.37 & $0.00038(4)$ & $0.00027(3)$ & $-0.00011(5)$ \\
7330.53 & 0.0 & $0.0539(14)$ & $0.0539(14)$ \\
\hline
\end{tabular}

\subsection{Radioactive Decay Data}

We have included gamma-rays from the radioactive decay of short-lived activation products in this evaluation. In most cases gamma-ray data from the ENSDF decay datasets were renormalized using the total cross section information from Mughabghab et al [10]. In some cases more recent cross sections from the literature were used for normalization. The Budapest data was used for normalization when the half-life correction was negligible and decay gamma rays were observed with better precision than the literature cross sections.

\subsection{Neutron Separation Energies and Total Cross Sections}

Byproducts of this work include neutron separation energies deduced from the level scheme and total neutron capture cross sections determined from 
the ground state feedings. For many light nuclei the capture gamma-ray data are nearly complete and the total primary gamma-ray cross section can also be used. In many cases decay gamma-rays could also be used to determine the total capture cross section. This was particularly applicable for determining the cross sections populating isomeric levels. Even if the data were incomplete, the total gamma-ray cross section feeding the ground state provides a lower limit for the total cross section. Table 2 gives a preliminary summary of the neutron separation energies and total neutron capture cross sections deduced here.

Notable discrepancies with respect to Mughabghab et al [10] total cross sections were observed for ${ }^{6} \mathrm{Li},{ }^{12} \mathrm{C},{ }^{33,34} \mathrm{~S}$, and ${ }^{37} \mathrm{Cl}$. These results are still under investigation.

\section{Final Results}

The capture gamma-ray library will be disseminated in several ways. An IAEA TECDOC will be published later this year. It will include complete prompt and decay gamma-ray tables, ordered by element and isotope, containing energy and $k_{0}$ values. Decay gamma-ray $k_{0}$ values must be corrected for saturation, and are presented assuming a 1 hour activation. Energyordered tables of the most intense gamma-rays will also be provided. There will also be a table of atomic and isotopic properties including abundance, total capture cross sections, and Westcott g-factors. In addition to the TECDOC, the database and its compete documentation will be provided on an accompanying CD-ROM. Dissemination software is being developed in collaboration with V. Zerkin of the IAEA to provide Internet access to the data with full gamma-ray searching capabilities.

A Handbook of Prompt Gamma-ray Activation Analysis is under development is association with Kluwer Scientific Publishers. This book will provide the gamma-ray energies, cross sections, and relative intensities as a percent of the strongest transition, for each isotope. Energy-ordered tables and atomic and isotopic properties will also be provided. An atlas of spectra for all elements will be included. The Handbook will provide detailed information on experimental PGAA analysis including the use of the library. An accompanying CD-ROM will also be included with the Handbook.

The ENSDF format datasets will be provided to the National Nuclear Data Center in Brookhaven for inclusion in the XUNDL experimental data library. Publication of this data in Nuclear Data Sheets is under consideration. The capture data will be available on the LBNL Isotopes Project's website at http://ie.lbl.gov/toi.html. 


\section{Acknowledgments}

This work was supported by the Director, Office of Science, Office of Basic

Energy Sciences, of the U.S. Department of Energy under Contract No. DEAC03-76SF00098 and by the Intenational Atomic Energy Agency, Vienna.

\section{References}

1. M.A. Lone, R.A. Leavitt and D.A. Harrison, Atom. Data Nucl. Data Tables 26, 511 (1981).

2. V.J. Orphan and N.C. Rasmussen, Nucl. Instrum. Meth. 48, 2 (1967).

3. Z. Revay, G. L. Molnar, T. Belgya, Z. Kasztovszky and R. B. Firestone, J. Radioanal. Nucl. Chem. bf 244, 383 (2000).

4. G. L. Molnr, Z. Rvay, T. Belgya and R. B. Firestone, Appl. Radiat. Isot. 53, 527 (2000).

5. Development of a Database for Prompt $\gamma$ - ray Neutron Activation Analysis, prepared by R. Paviotti-Corcuera and R.M. Lindstrom, INDC(NDS)-411 (1998).

6. Evaluated Nuclear Structure Data File, a computer file of evaluated experiimental nuclear structure data maintained by the National Nuclear Data Center, Brookhaven National Laboratory.

7. Nuclear Science Reference File, a bibliographic computer file of nuclear science references continually updated and maintained by the National Nuclear Data Center, Brookhaven National Laboratory. Recent literature scanned by D. Winchell.

8. R.C. Reedy and S.C. Frankle, Atom. Data Nucl. Data Tables 80, 511 (2002).

9. K.J.R. Rosman and P.D.P Taylor, Pure and Appl. Chem. 70, 217 (1998).

10. S.F. Mughabghab, M. Divadeenam, and N.E. Holden, Neutron Cross Sections, Vol. 1: Neutron Resonance Parameters and Thermal Cross Sections, Part A Z=1-60, Academic Press (1981); S.F. Mughabghab, Neutron Cross Sections, Vol. 1: Neutron Resonance Parameters and Thermal Cross Sections, Part B Z=61-100, Academic Press (1984).

11. N.E. Holden, Pure and Appl. Chem. 71, 2309 (1999).

12. IUPAC Commission on Atomic Weights and Isotopic Abundances, Atomic Weights of the Elements, Pure and Appl. Chem 68, 2339 (1996).

13. G. Audi and A.H. Wapstra, Nucl. Phys. A495, 409 (1995). 
Table 2. Neutron separation energies and total capture cross sections.

\begin{tabular}{|c|c|c|c|c|c|}
\hline Isotope & Abundance & $\mathrm{S}_{n}$ (Audi) & $\mathrm{S}_{n}$ (this work) & $\sigma$ (Mughabghab) & $\sigma($ this work $)$ \\
\hline${ }^{1} \mathrm{H}$ & $99.9885(70)$ & $2224.5725(22)$ & & $332.6(7) \mathrm{mb}$ & $332.6 \mathrm{mb}$ \\
\hline${ }^{2} \mathrm{H}$ & $0.0115(70)$ & $6257.2482(24)$ & & $0.519(7) \mathrm{mb}$ & $0.492(25) \mathrm{mb}$ \\
\hline${ }^{3} \mathrm{He}$ & $0.000137(3)$ & 20577.62 & & $0.031(9) \mathrm{mb}$ & \\
\hline${ }^{4} \mathrm{He}$ & $99.999863(3)$ & & & & \\
\hline${ }^{6} \mathrm{Li}$ & $7.59(4)$ & $7249.96(9)$ & $7249.94(4)$ & $39(3) \mathrm{mb}$ & $52.6(22) \mathrm{mb}$ \\
\hline${ }^{7} \mathrm{Li}$ & $92.41(4)$ & $2033.8(3)$ & $2032.57(4)$ & $45(3) \mathrm{mb}$ & $45.7(9) \mathrm{mb}$ \\
\hline${ }^{9} \mathrm{Be}$ & 100 & $6812.33(6)$ & $6812.10(3)$ & $7.6(8) \mathrm{mb}$ & $8.8(6) \mathrm{mb}$ \\
\hline${ }^{10} \mathrm{~B}$ & $19.9(7)$ & $11454.12(20)$ & $11454.15(14)$ & $500(200) \mathrm{mb}$ & $303(20) \mathrm{mb}$ \\
\hline${ }^{10} \mathrm{~B}(n, \alpha)$ & & & & $712.5(17) \mathrm{b}$ & $712.5 \mathrm{~b}$ \\
\hline${ }^{11} \mathrm{~B}$ & $80.1(7)$ & $3370.4(14)$ & & $6(3) \mathrm{mb}$ & \\
\hline${ }^{12} \mathrm{C}$ & $98.93(8)$ & $4946.310(10)$ & $4946.311(3)$ & $3.53(7) \mathrm{mb}$ & $3.89(6) \mathrm{mb}$ \\
\hline${ }^{13} \mathrm{C}$ & $1.07(8)$ & $8176.440(10)$ & $8176.61(18)$ & $1.37(4) \mathrm{mb}$ & $1.22(11) \mathrm{mb}$ \\
\hline${ }^{14} \mathrm{~N}$ & $99.632(7)$ & $10833.230(10)$ & $10833.317(12)$ & $75(8) \mathrm{mb}$ & $79.0(9) \mathrm{mb}$ \\
\hline${ }^{15} \mathrm{~N}$ & $0.368(7)$ & $2490.8(23)$ & & $24(8) \mathrm{mb}$ & \\
\hline${ }^{16} \mathrm{O}$ & $99.757(16)$ & $4143.33(21)$ & $4143.06(10)$ & $0.190(19) \mathrm{mb}$ & $0.189(8) \mathrm{mb}$ \\
\hline${ }^{17} \mathrm{O}$ & $0.038(1)$ & $8044.4(8)$ & & $0.54(7) \mathrm{mb}$ & \\
\hline${ }^{18} \mathrm{O}$ & $0.205(14)$ & $3957(3)$ & & $0.16(1) \mathrm{mb}$ & \\
\hline${ }^{19} \mathrm{~F}$ & 100 & $6601.31(5)$ & $6601.344(16)$ & $9.6(5) \mathrm{mb}$ & $9.50(11) \mathrm{mb}$ \\
\hline${ }^{20} \mathrm{Ne}$ & $90.48(3)$ & $6761.11(4)$ & $6761.19(5)$ & $37(4) \mathrm{mb}$ & \\
\hline${ }^{21} \mathrm{Ne}$ & $0.27(1)$ & & & $670(110) \mathrm{mb}$ & \\
\hline${ }^{22} \mathrm{Ne}$ & $9.25(3)$ & $5200.62(12)$ & $5200.64(17)$ & $45(6) \mathrm{mb}$ & \\
\hline${ }^{23} \mathrm{Na}$ & 100 & $6959.44(5)$ & $6959.592(15)$ & $530(5) \mathrm{mb}$ & $527(7) \mathrm{mb}$ \\
\hline${ }^{24} \mathrm{Mg}$ & $78.99(4)$ & $7330.67(4)$ & $7330.53(4)$ & $51(5) \mathrm{mb}$ & $53.7(14) \mathrm{mb}$ \\
\hline${ }^{25} \mathrm{Mg}$ & $10.00(1)$ & 11093.09(4) & $11093.157(21)$ & $190(30) \mathrm{mb}$ & $193(2) \mathrm{mb}$ \\
\hline${ }^{26} \mathrm{Mg}$ & $11.01(3)$ & $6443.35(4)$ & $6443.35(3)$ & $38.2(8) \mathrm{mb}$ & $37.7(13) \mathrm{mb}$ \\
\hline${ }^{27} \mathrm{Al}$ & 100 & $7725.05(6)$ & $7725.170(4)$ & $231(3) \mathrm{mb}$ & $232(3) \mathrm{mb}$ \\
\hline${ }^{28} \mathrm{Si}$ & $92.2297(7)$ & $8473.56(3)$ & $8473.537(23)$ & $177(5) \mathrm{mb}$ & $186(3) \mathrm{mb}$ \\
\hline${ }^{29} \mathrm{Si}$ & $4.6832(5)$ & $10609.18(3)$ & 10609.23(3) & $101(14) \mathrm{mb}$ & $118(3) \mathrm{mb}$ \\
\hline${ }^{30} \mathrm{Si}$ & $3.0872(5)$ & $6587.40(5)$ & $6587.39(3)$ & $107.0(20) \mathrm{mb}$ & $116(3) \mathrm{mb}$ \\
\hline${ }^{31} \mathrm{P}$ & 100 & $7935.65(4)$ & $7935.596(23)$ & $172(6) \mathrm{mb}$ & $167(5) \mathrm{mb}$ \\
\hline${ }^{32} \mathrm{~S}$ & $94.93(31)$ & $08641.58(3)$ & $8641.809(25)$ & $530(40) \mathrm{mb}$ & $536(8) \mathrm{mb}$ \\
\hline${ }^{33} \mathrm{~S}$ & $0.76(2)$ & $11416.94(5)$ & $11417.219(16)$ & $350(40) \mathrm{mb}$ & $461(15) \mathrm{mb}$ \\
\hline${ }^{34} \mathrm{~S}$ & $4.29(28)$ & $6985.84(4)$ & $6986.091(15)$ & $227(5) \mathrm{mb}$ & $277(8) \mathrm{mb}$ \\
\hline${ }^{36} \mathrm{~S}$ & $0.02(1)$ & $4303.58(9)$ & $4303.608(25)$ & $150(30) \mathrm{mb}$ & \\
\hline${ }^{35} \mathrm{Cl}$ & $75.78(4)$ & $8579.70(7)$ & $8579.672(18)$ & $43.6(4) \mathrm{b}$ & $44(3) \mathrm{b}$ \\
\hline${ }^{37} \mathrm{Cl}$ & $24.22(4)$ & $6107.78(10)$ & $6107.73(9)$ & $433(6) \mathrm{mb}$ & $553(23) \mathrm{mb}$ \\
\hline
\end{tabular}

